\title{
Resistência de união e degradação de um compósito fluido autoaderente em substratos dentais e um compósito restaurador
}

\section{Bond strength and degradation of a self-adhesive flowable composite in dental substrates and a restorative composite}

\author{
Rubens Nazareno Garcia* \\ Ana Paula de Lucca Werlich ${ }^{* *}$ \\ Bárbara Pamela Siqueira da Costa** \\ Flávia Carvalho Gonçalves** \\ Juliani Rafaeli* \\ Priscila Carla Dambros ${ }^{* *}$
}

\section{Resumo}

Objetivo: avaliar a resistência de união $(R U)$ de um compósito autoaderente em quatro substratos usados na técnica direta, nos períodos de uma semana e de um ano de armazenamento em água destilada. Materiais e método: foram utilizadas 96 amostras (5 $\mathrm{mm}$ largura / $15 \mathrm{~mm}$ comprimento / $2 \mathrm{~mm}$ espessura) de dentes bovinos - esmalte hígido (EH), esmalte desgastado (ED), dentina média (DM) - e do compósito restaurador direto $(C D)$ Filtek $Z 350 X T^{R}$ (3M ESPE), sendo 24 amostras por substrato. As amostras foram divididas em dois grupos, conforme o período de armazenamento. Foram utilizados dois compósitos fluidos: o controle Filtek Z350XT Flow ${ }^{R}$ (3M ESPE) (FF) e o autoaderente Dyad Flow $^{R} /$ Kerr (DF), aplicados aos quatro substratos $(n=6)$. Em cada amostra, foram posicionadas quatro matrizes cilíndricas transparentes, preenchidas com os compósitos FF/DF, e fotopolimerizadas por vinte segundos. As matrizes foram removidas para expor os corpos de prova (24 por grupo) e armazenadas em água destilada $37 \pm 2^{\circ} \mathrm{C}$ por dois períodos, uma semana e um ano. Após cada período, os corpos de prova foram submetidos ao ensaio de resistência de união ao cisalhamento (velocidade de $1,0 \mathrm{~mm} / \mathrm{min}$ ). Os resultados foram analisados pelo ANOVA/teste de Tukey $(p<0,05)$. Resultados: nos ensaios de uma semana, $O D F, o E D$ e o $C D$ apresen- taram maior $R U$, não diferindo entre si. O EH e o DM apresentaram menor $R U$, sem diferença significativa. Nos ensaios de um ano, com o DF, o comportamento foi similar, porém com menor RU para o EH e o DM. Conclusão: os resultados mostram que o compósito Dyad Flow ${ }^{R}$ após um ano de armazenamento em água destilada, apresentou resistência de união superior ao cisalhamento nos substratos esmalte desgastado e compósito direto.

Palavras-chave: Dentina. Esmalte dentário. Resinas compostas. Resistência ao cisalhamento.

\section{Introdução}

A introdução da técnica do condicionamento ácido do esmalte por Buonocore ${ }^{1}$, em 1955, criou nova perspectiva nos procedimentos restauradores, dando início à Odontologia Adesiva. Ainda que os sistemas adesivos convencionais sejam largamente utilizados ${ }^{2}$, os adesivos autocondicionantes permitem procedimentos clínicos mais simples, rápidos e

Professor doutor do curso de Odontologia, Universidade do Vale do Itajaí, SC, Brasil - Grupo de Pesquisa Biomateriais em Odontologia e departamento de Odontologia, Universidade da Região de Joinville, SC, Brasil.

Acadêmica do curso de Odontologia, Universidade do Vale do Itajaí, SC, Brasil. 
com menor sensibilidade envolvendo a técnica ${ }^{3}$. A ação desses produtos no substrato dental envolve a simultânea desmineralização da estrutura dental e a infiltração na mesma profundidade, garantindo a completa penetração do adesivo ${ }^{4}$, além da interação química entre os monômeros funcionais e a hidroxapatita residual ${ }^{5}$.

Os compósitos fluidos foram, primeiramente, introduzidos no mercado em 1995, para restaurações de lesões Classe V. De acordo com Bayne et al. ${ }^{6}$ (1998), eles apresentam excelentes propriedades de manuseio por terem baixa viscosidade e reduzem o tempo de trabalho dos clínicos. Seguindo as mesmas características, foi, recentemente, introduzido no mercado o compósito autoaderente Vertise Flow $^{\mathrm{R}}$ (Kerr, Orange, CA, USA - chamado Dyad Flow na América Latina), assim como o Fusio (Pentron Clinical, Orange, CA, USA) e o Constic ${ }^{\mathrm{R}}$ (DMG America, Englewood, NJ, USA). Esses compósitos não necessitam da aplicação prévia de um sistema adesivo e são requeridos por contar com a interação química e micromecânica entre o material e a estrutura dental ou outros substratos, alcançadas com a incorporação de um monômero ácido ${ }^{7-9}$.

O objetivo deste estudo foi avaliar a resistência de união (RU) de um compósito autoaderente em quatro substratos usados na técnica direta, com uma semana e com um ano de armazenamento em água destilada. A hipótese nula testada foi de que as resistências de união do compósito fluido autoaderente seriam estatisticamente similares quando comparados os tempos de armazenamento.

\section{Materiais e método}

Foram utilizadas 96 amostras ( $5 \mathrm{~mm}$ largura, $15 \mathrm{~mm}$ comprimento e $2 \mathrm{~mm}$ espessura) de dentes bovinos. As raízes dos dentes bovinos foram removidas com o auxílio de um disco flexível diamantado (KG Sorensen ${ }^{\mathrm{R}}$, Cotia, SP, Brazil) sob refrigeração. As coroas foram seccionadas, utilizando o mesmo disco diamantado e também sob refrigeração, para obter 24 amostras de esmalte hígido/EH, 24 amostras de esmalte desgastado/ED (abrasionados com lixa d'água, granulações 200 e 600) e 24 amostras de superfícies planas de dentina média DM (1 mm abaixo da junção amelodentinária). Anteriormente aos procedimentos adesivos, as amostras de dentina foram abrasionadas com lixa d'água 600 para criar lama dentinária. Ainda, 24 blocos do compósito restaurador direto Filtek Z350 XT ${ }^{\mathrm{R}} / \mathrm{CD}$ (3M ESPE) foram preparados na mesma dimensão das amostras dos dentes bovinos.

As amostras de cada substrato foram divididas em dois grupos para cada período de armazenamento $(n=6)$, e os produtos foram utilizados de acordo com as instruções dos fabricantes. Dois compósitos fluidos foram aplicados aos quatro substratos: o controle Filtek Z350 XT Flow ${ }^{\mathrm{R}}$ (3M ESPE) (FF) e o autoaderente Dyad Flow ${ }^{\mathrm{R}} / \mathrm{Kerr}$ (DF) (Quadro 1). Para o Filtek Z350 XT Flow ${ }^{\mathrm{R}}$, o protocolo adesivo envolveu a aplicação do ácido fosfórico por 30 segundos no esmalte hígido e desgastado (e também nos blocos do compósito Filtek Z350 XT ${ }^{\mathrm{R}}$ ) e 15 segundos na dentina, lavagem, secagem 5 segundos, aplicação do adesivo, ar 5 segundos e fotopolimerização por 10 segundos. No Dyad Flow ${ }^{\mathrm{R}}$, o protocolo adesivo envolveu a aplicação do ácido fosfórico por 30 segundos no esmalte hígido e desgastado e no compósito Filtek $\mathrm{Z} 350 \mathrm{XT}^{\mathrm{R}}$. Esse tratamento não ocorreu na dentina, conforme recomendações do fabricante. 


\begin{tabular}{|c|c|c|c|}
\hline Materiais & Lote & Composições & Protocolos \\
\hline $\begin{array}{l}\text { Acid Gel }^{R} \\
\text { Dentalville do Brasil Joinville, } \\
\text { SC, Brasil } \\
\text { Sistema adesivo } \\
\text { Adper Single Bond } 2^{R} \\
p H \approx 4.7 \\
3 M \text { ESPE - St. Paul, MN, USA }\end{array}$ & N2I1104BR & $\begin{array}{l}\text { Ácido fosfórico } 37 \% \text { e clorexidina } 2 \% \\
\text { Bis-GMA, HEMA, UDMA, dimetacrilatos, } \\
\text { etanol, água, canforquinona, fotoiniciadores, } \\
\text { copolímero do ácido polialcenóico, sílica ( } 5 \\
\text { nm) }\end{array}$ & $\begin{array}{l}\text { Aplicar o ácido fosfóri- } \\
\text { co } 30 \text { s para o esmalte } \\
\text { e } 15 \text { s para a dentina, } \\
\text { lavar, secar } 5 \text { s, aplicar } \\
\text { o adesivo, ar } 5 \text { s, foto } \\
10 \text { s. }\end{array}$ \\
\hline $\begin{array}{l}\text { Filtek Z350 XT Flow } \\
\text { Nanocompósito } \\
\text { fluido/A2 } \\
3 \mathrm{M} \text { ESPE - St. Paul, MN, USA }\end{array}$ & 1211700713 & $\begin{array}{l}\text { Bis-GMA, TEGDMA, Bis-EMA, cerâmica } \\
\text { tratada com silano, sílica, óxido de zircônio } \\
-55 \% \text { vol / } 65 \% \text { peso }\end{array}$ & Aplicar e foto 20s. \\
\hline $\begin{array}{l}\text { Dyad Flow } \\
\text { Nanocompósito fluido } \\
\text { autoaderente/A2 } \\
\mathrm{pH} \approx 1.9 \text { (antes foto) } \\
\mathrm{pH} \approx 6.5-7 \text { (após foto) } \\
\text { Kerr, Orange, CA, USA }\end{array}$ & 4398621 & $\begin{array}{l}\text { GPDM, partículas pré-polimerizadas, par- } \\
\text { tículas de vidro de bário com } 1-\mu \mathrm{m} \text {, na- } \\
\text { nopartículas de sílica coloidal, fluoreto de } \\
\text { itérbium nanoparticulado }\end{array}$ & $\begin{array}{l}\text { Aplicar, esfregar uma } \\
\text { fina camada }(<0,5 \mathrm{~mm}) \\
\text { com pressão por } 15- \\
20 \mathrm{~s} \text {, foto } 20 \mathrm{~s} \text {. }\end{array}$ \\
\hline $\begin{array}{l}\text { Filtek } Z 350 X^{R}{ }^{R} \\
\text { Nanocompósito restaurador/A2B } \\
3 \text { M ESPE } \\
\text { St. Paul, MN, USA }\end{array}$ & N312081BR & $\begin{array}{l}\text { Bis-GMA, UDMA, TEGDMA, e Bis-EMA, } \\
\text { partículas de sílica não aglomeradas e não } \\
\text { agregadas } 20 \mathrm{~nm} \text {, partículas de zircônia não } \\
\text { aglomeradas e não agregadas } 4 \text { a } 11 \mathrm{~nm}, \\
\text { e partículas de sílica/zircônia agregadas - } \\
63,3 \% \text { vol, 78,5\% peso }\end{array}$ & Aplicar e foto $20 \mathrm{~s}$. \\
\hline
\end{tabular}

Abreviações conforme fabricantes: Bis-GMA, bisphenol glycidyl dimethacrylate; HEMA, 2-hydroxy ethyl methacrylate; UDMA, urethane dimethacrylate; TEGDMA, triethylene glycol dimethacrylate; Bis-EMA, bisphenol A polyethylene glycol dimethacrylate; GPDM, glycerol phosphate dimethacrylate.

Fonte: dados da pesquisa.

De acordo com Shimada et al. ${ }^{10}$ (2002), para todos os grupos e em cada amostra, quatro matrizes cilíndricas (Tygon tubing - TYG-030, Saint-Gobain Performance Plastic, Maime Lakes, FL, USA) foram posicionadas, as quais foram preenchidas com os compósitos $\mathrm{FF}$ e DF e fotopolimerizadas por 20 segundos (LED Bluephase ${ }^{\mathrm{R}}-1.200 \mathrm{~mW} / \mathrm{cm}^{2}-$ Ivoclar Vivadent, Schaan, Principado de Liechtenstein). As matrizes cilíndricas foram removidas para expor os corpos de prova (24 cilindros por grupo com área de $0,38 \mathrm{~mm}^{2}$ pela fórmula $\omega \mathrm{R}^{2}$ ) e as amostras armazenadas em água destilada a $37 \pm 2^{\circ} \mathrm{C}$ por uma semana e por um ano (com trocas semanais de água). Após cada período, as amostras foram acopladas a uma máquina de ensaios universal (EMIC DL $1000^{\mathrm{R}}$, São José dos Pinhais, PR, Brasil) e os corpos de prova submetidos ao ensaio de resistência de união ao cisalhamento, aplicado na base dos corpos de prova com um fio ortodôntico fino/ $0,25 \mathrm{~mm}$ - a uma velocidade de $1,0 \mathrm{~mm} / \mathrm{min}$ - até o rompimento da união (Figura 1).

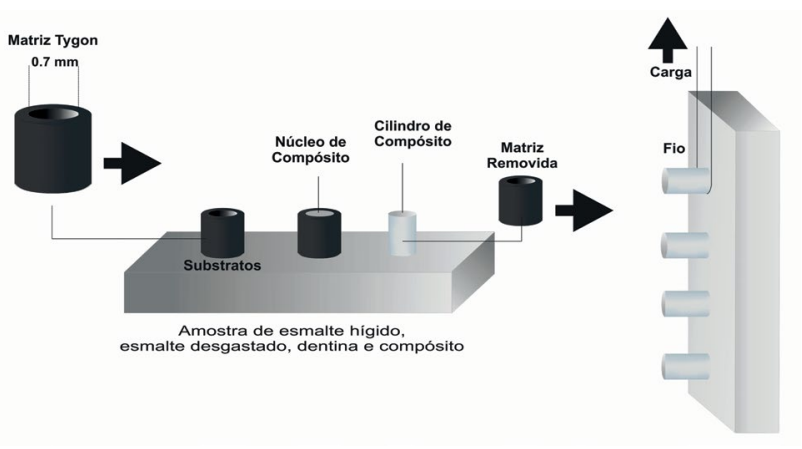

Figura 1 - Representação esquemática da metodologia empregada Fonte: elaboração dos autores.

Foi utilizada microscopia óptica para avaliação das fraturas/falhas, que foram classificadas em adesivas, mistas (adesivas e coesivas) e coesivas nos substratos. Os resultados foram analisados pela Análise de Variância/ANOVA multifatorial (dois compósitos fluidos, quatro substratos e dois tempos) e pelo teste de Tukey $(p<0,05)$. 


\section{Resultados}

A análise de variância ANOVA multifatorial mostrou diferenças significativas entre os compósitos fluidos, substratos e tempos $(p<0,05)$. Para identificar as diferenças foi aplicado o teste de Tukey $(\mathrm{p}<0,05)$.

Nos ensaios de uma semana com o produto controle Filtek Flow $^{\mathrm{R}}$, somente o esmalte hígido resultou em menor resistência de união, ainda que sem diferença significativa para o esmalte desgastado. Para o Dyad Flow ${ }^{\mathrm{R}}$ o esmalte desgastado e o compósito direto apresentaram maior resistência de união, não diferindo entre si. Comparando os compósitos Flow em cada substrato, não houve diferença estatística para o esmalte hígido e para 0 compósito direto, porém, o Dyad Flow ${ }^{\mathrm{R}}$ apresentou maior resistência de união em esmalte desgastado. O mesmo não ocorreu na dentina média, pois o Filtek Flow ${ }^{\mathrm{R}}$ resultou em maior resistência de união (Tabela 1).

Tabela 1 - Médias de RU ( $\pm D P)$ em MPa e teste de Tukey $(p<0,05)$ uma semana

\begin{tabular}{|c|c|c|}
\hline \multirow{2}{*}{ Uma semana } & \multicolumn{2}{|c|}{ Compósito } \\
\hline & Filtek Flow & Dyad Flow \\
\hline Esmalte hígido & $20,8 \pm 1,1 \mathrm{~A} \quad \mathrm{~b}$ & $24,0 \pm 3,1 \quad A$ \\
\hline Esmalte desgastado & $22,7 \pm 3,8$ B a b & $29,7 \pm 5,5 \quad A$ \\
\hline Dentina média & $24,9 \pm 4,6 \mathrm{~A} \quad \mathrm{a}$ & $20,8 \pm 3,2 \quad B$ \\
\hline Compósito direto & $25,9 \pm 6,2 \mathrm{~A} \quad \mathrm{a}$ & $28,4 \pm 5,1 \quad A$ \\
\hline
\end{tabular}

Médias seguidas da mesma letra maiúscula nas linhas e da mesma letra minúscula nas colunas não diferem significativamente entre si pelo teste de Tukey $(p<0,05)$.

Fonte: dados da pesquisa
Nos ensaios de um ano com o produto controle Filtek Flow ${ }^{\mathrm{R}}$, somente o compósito direto resultou em maior resistência de união, e a dentina média mostrou a menor resistência de união entre todos os substratos, com diferença estatística. Para o Dyad Flow ${ }^{\mathrm{R}}$, o esmalte desgastado e o compósito direto apresentaram maior resistência de união, não diferindo entre si. Comparando os dois compósitos Flow em cada substrato, somente o substrato esmalte desgastado não apresentou decréscimo na resistência de união após um ano de armazenamento em água (Tabelas 2 e 3 ).

Tabela 2 - Médias de RU ( $\pm D P)$ em MPa e teste de Tukey $(p<0,05)$ um ano

\begin{tabular}{l|cc|ccc}
\hline \multirow{2}{*}{\multicolumn{1}{c|}{ Um ano }} & \multicolumn{5}{c}{ Compósito } \\
\cline { 2 - 6 } & \multicolumn{2}{|c}{ Filtek Flow } & \multicolumn{3}{c}{ Dyad Flow } \\
\hline Esmalte hígido & $24,2 \pm 7,3 \mathrm{~A}$ & $\mathrm{~b}$ & $13,4 \pm 6,2$ & $\mathrm{~B}$ & $\mathrm{~b}$ \\
Esmalte desgastado & $24,5 \pm 3,7 \mathrm{~A}$ & $\mathrm{~b}$ & $25,6 \pm 5,2$ & $\mathrm{~A}$ & $\mathrm{a}$ \\
Dentina média & $18,6 \pm 4,4 \mathrm{~A}$ & $\mathrm{c}$ & $12,9 \pm 4,1$ & $\mathrm{~B}$ & $\mathrm{~b}$ \\
Compósito direto & $30,1 \pm 4,7 \mathrm{~A}$ & a & $24,3 \pm 8,2$ & $\mathrm{~B}$ & $\mathrm{a}$
\end{tabular}

Médias seguidas da mesma letra maiúscula nas linhas e da mesma letra minúscula nas colunas não diferem significativamente entre si pelo teste de Tukey $(p<0,05)$.

Fonte: dados da pesquisa.

Comparando os resultados para os tempos de uma semana e de um ano, para o Filtek Flow ${ }^{\mathrm{R}}$, não houve diferença estatística na resistência de união para todos os substratos. No entanto, para o Dyad Flow ${ }^{\mathrm{R}}$, houve um decréscimo na resistência de união nos substratos esmalte hígido e dentina média, com diferença significativa para o esmalte desgastado e o compósito direto, que apresentaram maior resistência de união (Tabela 3).

Tabela 3 - Médias de RU ( \pm DP) em MPa e teste de Tukey $(p<0,05)$ - nos 2 tempos

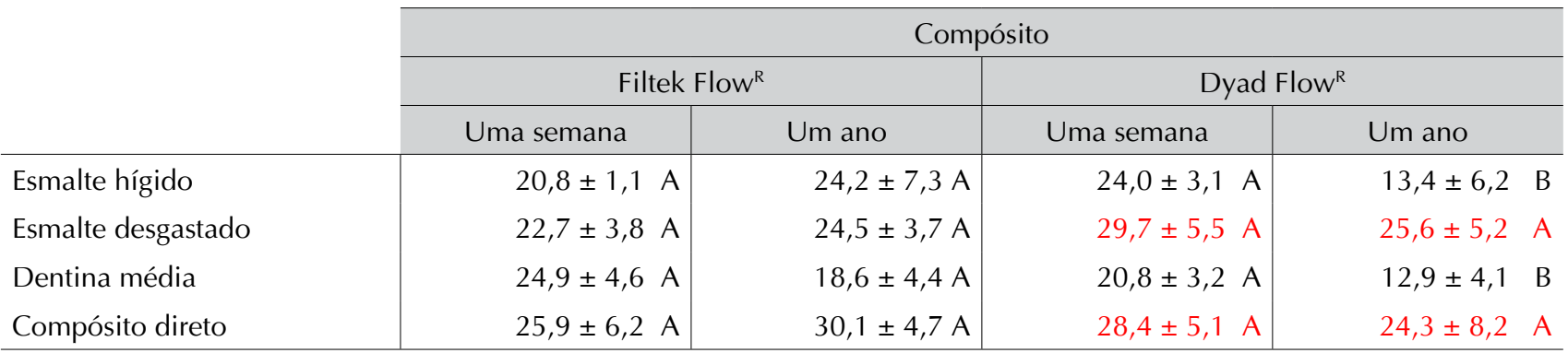

Anos dentro de cada produto: médias seguidas da mesma letra maiúscula nas linhas, dentro de cada produto, não diferem significativamente entre si pelo teste de Tukey $(p<0,05)$.

Fonte: dados da pesquisa.

Na microscopia óptica, foram observadas falhas mistas para todos os grupos, incluindo, principalmente, falhas coesivas nos substratos, na transição da falha adesiva para coesiva. Esse tipo de fratura é, habitualmente, observada nos ensaios de cisalhamento.

\section{Discussão}

Com base nos achados do presente estudo, a hipótese nula formulada não deve ser rejeitada para o esmalte desgastado e o compósito direto. No entanto, deve ser rejeitada para o esmalte hígido e para a dentina média, porque os resultados mostraram diferenças estatisticamente significativas nas resis- 
tências de união ao cisalhamento, com um ano de armazenamento em água destilada.

Embora ensaios clínicos produzam evidências mais confiáveis e a tradução dos resultados in vitro para as condições bucais apresentem limitações, os testes laboratoriais são úteis em produzir informações mais rápidas que norteiam o uso clínico dos materiais odontológicos. Especificamente, ensaios de resistência de união têm sido considerados por fornecer uma avaliação quantitativa da união e/ ou adesão dos materiais, baseados no conceito de que quanto maior a união e/ou adesão, mais o material irá resistir ao estresse de contração e funcional. Dentre eles, os mais efetivos são os ensaios de microtração, largamente utilizados e responsáveis pelos resultados mais confiáveis em termos de resistência de união. No entanto, pela facilidade de obtenção dos corpos de prova nos ensaios de cisalhamento e microcisalhamento, eles têm sido também muito utilizados para testes mecânicos dos materiais adesivos ${ }^{3,11}$.

A presente pesquisa avaliou a resistência de união ao microcisalhamento de dois compósitos fluidos, o controle Filtek Flow ${ }^{\mathrm{R}}$ e o autoaderente Dyad Flow $^{\mathrm{R}}$, com foco nesse último, por ser uma nova classe de material adesivo. Os compósitos fluidos foram aderidos a quatro substratos utilizados na técnica direta, utilizando a metodologia do microcisalhamento proposta por Shimada et al. ${ }^{10}$ (2002), em dois períodos de tempo: uma semana e um ano. Esse tipo de ensaio mecânico soluciona problemas relacionados à propagação das tensões na interface de união em áreas maiores. Apresenta a vantagem de que vários corpos de prova podem ser obtidos a partir de uma amostra, sendo mais fácil e mais barato do que os testes de microtração $0^{8,9}$, nos quais as amostras devem ser cortadas para que sejam obtidos os corpos de prova ${ }^{12}$.

Como o mecanismo de ação dos sistemas adesivos autocondicionantes é similar aos compósitos fluidos autoaderentes, ou seja, ocorre pela ação simultânea dos monômeros mais ácidos nos substratos, parece pertinente discutir os resultados obtidos com os compósitos aderentes na mesma linha de raciocínio. A adesão à superfície do esmalte é baseada na infiltração dos monômeros resinosos ao esmalte condicionado, e um ótimo padrão de condicionamento é observado quando se utiliza a técnica convencional de condicionamento utilizando o ácido fosfórico. Adicionalmente, os sistemas adesivos autocondicionantes também têm sido preconizados para esse tipo de procedimento como um substituto adequado para os sistemas convencionais ${ }^{13}$.

Contudo, ainda existe alguma preocupação com relação à habilidade desses adesivos autocondicionantes em condicionar o esmalte. Muitos estudos mostraram que a resistência de união do compósito ao esmalte, fornecida por adesivos ultrassuaves, suaves ou intermediários, é menor quando comparada aos sistemas convencionais ${ }^{14-17}$. Porém, os sistemas autocondicionantes parecem ter um bom desempenho em estudos clínicos ${ }^{18}$, e o condicionamento seletivo do esmalte com ácido fosfórico demonstrou ser uma técnica de excelência se utilizada com os sistemas autocondicionantes, melhorando a união ao esmalte es-21. $^{\text {. }}$

Para este estudo, de acordo com as instruções dos fabricantes, o esmalte hígido e o esmalte desgastado foram condicionados com ácido fosfórico nas amostras do grupo controle e, adicionalmente, condicionados com ácido fosfórico nos grupos DF. De acordo com Van Meerbeek et al. ${ }^{3}$ (2003), o condicionamento ácido prévio do esmalte melhora significativamente a efetividade da união, uma vez que o ácido fosfórico aumenta a energia de superfície do esmalte e proporciona mais microrretenção ${ }^{22}$.

Conforme pode ser observado nas Tabelas 1 e 2 , para o produto DF, houve diferença estatística entre o esmalte hígido e o esmalte desgastado, com maior resistência de união para o esmalte desgastado, para ambos os tempos. Di Hipólito ${ }^{23}$ (2005) descreve o mecanismo de ação dos monômeros resinosos com o esmalte, que é devido a uma sequência de fenômenos interdependentes. Possivelmente, fatores como o aumento dos espaços inter e intraprismáticos para a infiltração dos monômeros resinosos, quebrando a tensão superficial do monômero líquido e a formação de um ângulo de contato menor com a superfície do esmalte, são responsáveis pela maior resistência de união ao esmalte desgastado. Ainda, a exposição da camada prismática pode ter favorecido a qualidade do processo de desmineralização e a infiltração do compósito fluido DF. A Tabela 3 mostra que não houve significativo decréscimo de união para o esmalte desgastado com um ano de armazenamento em água destilada.

Baseado no pH 1,9 declarado pelo fabricante (antes da fotopolimerização), é possível dizer que o Dyad Flow ${ }^{\mathrm{R}}$ pode interagir de forma semelhante a um sistema adesivo autocondicionante suave, sendo suficiente para desmineralizar, principalmente, o esmalte desgastado e interagir de forma similar também com o compósito restaurador Filtek Z350 $\mathrm{XT}^{\mathrm{R}}$ (para ensaios de uma semana, de um ano e para a comparação dos tempos). O Dyad Flow tem um elemento em comum com todos os agentes de união do fabricante Kerr, que é o monômero adesivo GPDM (glicerol fosfato dimetacrilato), um grupo fosfato funcional que cria uma interação química com os íons de cálcio da estrutura dental.

Os monômeros GPDM garantem uma união efetiva ao esmalte e à dentina, evidenciada pela força conhecida de todas as gerações dos adesivos OptiBond/Kerr. Atuando como um agente de união, de um lado, há um grupo fosfato acidulado para condicionar a estrutura dental e, ainda, interagir quimicamente com os íons de cálcio presentes na estrutura dental; de outro, há dois grupos funcionais de metacrilato para a copolimerização com outro monômero metacrilato, para promover uma densidade 
de ligações cruzadas aumentada e uma maior resistência mecânica para o adesivo polimerizado, mesma razão para a maior resistência de união apresentada para o substrato nanocompósito direto.

A dentina, por sua vez, é um substrato muito mais heterogêneo e fisiologicamente mais dinâmico do que o esmalte. Garberoglio e Brännström ${ }^{24}$ (1976) mostraram que o número e o diâmetro dos túbulos dentinários aumentam com a profundidade. Na dentina superficial, $96 \%$ da área é ocupada pela dentina intertubular, $3 \%$ pela dentina peritubular e apenas $1 \%$ pelo fluido dentinário. Existe uma relação inversa, no entanto, para a área mais próxima da polpa, de modo que $66 \%$ da área é ocupada por dentina peritubular, $12 \%$ por dentina intertubular e $22 \%$ por fluido dentinário. De acordo com Swift Junior et al. ${ }^{25}$ (1995), diferenças na composição e morfologia com relação à profundidade podem influenciar diretamente no comportamento e nas propriedades mecânicas da dentina contra agentes químicos e físicos aos quais a dentina é submetida durante os procedimentos operatórios, assim como a aplicação do compósito autoaderente utilizado no presente estudo.

O Dyad Flow ${ }^{\mathrm{R}}$ apresentou menor resistência de união à dentina média com um ano de armazenamento em água destilada. Entre as possíveis razões para tal resultado, a molhabilidade do material deve ser considerada. A adequada molhabilidade de um material adesivo em um substrato permite uma interação mais próxima entre o adesivo e o substrato. Possivelmente, essa propriedade não ocorreu de forma tão eficiente entre o DF e a dentina média ${ }^{7}$.

Para alguns autores ${ }^{8,26,27}$, como os componentes dos adesivos autocondicionantes de um frasco apresentam propriedades de hidrofilicidade e hidrofobicidade antagônicas, eles formam uma camada híbrida com infiltração incompleta no substrato dentinário, e isso, provavelmente, também ocorre, de forma mais pronunciada, em moléculas tão complexas como as do produto DF - que ao mesmo tempo funcionam como ácido, primer, adesivo e compósito fluido propriamente dito. Esses autores relataram que as camadas híbridas de alguns materiais exibiam canais microscópicos preenchidos por água, que permitiam o movimento dessa água na interface dentina-compósito. A redução da resistência de união à dentina, enfim, pode ser atribuída à degradação das fibrilas colágenas e/ou da resina adesiva, pelo processo de cisão da cadeia polimérica durante o qual essa cadeia é quebrada em segmentos menores, chamados de oligômeros.

Apesar da descrição do fabricante (Kerr), não existem dados disponíveis sobre a análise química do potencial de união do GPDM. Isso indica que o GPDM condiciona, ao invés de se unir a hidroxiapatita $^{5}$. Para alcançar a autoaderência, especula-se que um compósito relativamente viscoso (fluido) deva conter um monômero funcional que possua um potencial efetivo de interação química, já que não pode penetrar profundamente no substrato ${ }^{23}$, como ocorre com os sistemas adesivos. De qualquer forma, os achados do presente estudo estão de acordo com o que foi encontrado por Bektas et al. ${ }^{28}$ (2013), em relação à dentina e à metodologia da resistência de união ao microcisalhamento. Para Bektas et al., o Vertise Flow ${ }^{\mathrm{R}}$ (similar ao Dyad Flow) também apresentou menor resistência de união, quando comparado ao grupo controle.

De acordo com os achados de Teixeira et al. ${ }^{29}$ (2005), três mecanismos de interação química são possíveis para a resistência de união dos compósitos fluidos ao compósito restaurador direto, a saber: 1) a adesão entre as matrizes poliméricas, dos compósitos fluidos e do compósito restaurador; 2) a adesão entre as partículas de carga expostas de ambos os compósitos; 3) a formação de um microentrelaçamento das cadeias poliméricas e das partículas de carga de ambos os compósitos. Esse último mecanismo, provavelmente, domina e produz maior contribuição com relação a uma RU aceitável, como foi possível observar dentro do grupo controle e do grupo do compósito autoaderente DF e, ainda, entre os grupos, sem diferenças estatísticas entre eles.

Utilizando microscopia óptica, foi observado que os ensaios de RU ao cisalhamento tendem a produzir falhas coesivas do substrato ${ }^{8,9,26,27}$. A melhora nas propriedades dos materiais restauradores tem aumentado a RU e modificado os padrões de falhas ${ }^{30}$. Essa transição está relacionada à mudança no padrão de estresse à medida que a falha progride ao longo da interface. A maior parte de fraturas coesivas foi observada no substrato (quando o substrato é puxado para fora), na transição de falha adesiva para coesiva ${ }^{11}$. Pela microscopia óptica, em acordo com os autores citados, também foram observadas falhas mistas para todos os grupos, incluindo, principalmente, falhas coesivas nos substratos.

\section{Conclusão}

Os resultados mostraram que o Filtek Flow ${ }^{\mathrm{R}}$, após um ano de armazenamento em água destilada, apresentou resistências de união similares em todos os substratos estudados. Para o Dyad Flow ${ }^{\mathrm{R}}$, houve um decréscimo nas resistências de união dos substratos esmalte hígido e dentina média, com diferenças significativas para o esmalte desgastado e o compósito direto. Mais estudos são necessários para o entendimento sobre o mecanismo de ação e longevidade dos compósitos fluidos autoaderentes. 


\section{Abstract}

Objective: This study aimed to assess the shear bond strength (SBS) of a self-adhesive flowable composite in four substrates used in the direct technique, with one week and one year of storage in distilled water. Materials and method: Ninety-six samples (5-mm wide / 15-mm long / 2-mm thick) of bovine teeth - uncut enamel, cut enamel, medium dentin - and direct restorative composite/CD Filtek Z350XT TM (3M ESPE) were used; there were 24 samples per substrate. The samples were divided into two groups according to storage time. Two flowable composites (Control/FF - Filtek Z350 $X T$ Flow ${ }^{T M} / 3 M$ ESPE, and the self-adhesive/DF - Dyad Flow ${ }^{T M} /$ Kerr) were bonded to the four substrates $(n=6)$. Four tubings were positioned on each sample, filled with FF/DF composites, and light-cured for $20 \mathrm{~s}$. The tubings were removed to expose the specimens (24 per group) and stored in distilled water at $37 \pm 2^{\circ} \mathrm{C}$ for one week and one year. After each period, the specimens were subjected to the Shear Bond Strength (SBS) test (speed of $1.0 \mathrm{~mm} / \mathrm{min}$ ). The results were analyzed by Anova and Tukey's test ( $p<0.05)$. Results: For the one-week tests with DF composite, cut enamel and direct restorative composite showed higher SBS, with no difference between themselves. Uncut enamel and medium dentin presented lower SBS, with no significant difference. For the one-year test with DF composite, the behavior was similar, however uncut enamel and medium dentin showed lower SBS. Conclusion: The results show that Dyad Flow ${ }^{T M}$ composite, after one year of storage in distilled water, showed higher shear bond strength in cut enamel and direct composite substrates.

Keywords: Composite resins. Dental enamel. Dentin. Shear strength.

\section{Referências}

1. Buonocore MG. A simple method of increasing the adhesion of acrylic filling materials to enamel surfaces. J Dent Res $1955 ; 34(6): 849-53$

2. Juloski J, Goracci C, Rengo C, Giovannetti A, Vichi A, Vulicevic ZR, et al. Enamel and dentin bond strength of new simplified adhesive materials with and without preliminary phosphoric acid-etching. Am J Dent 2012; 25(4):239-43.

3. Van Meerbeek B, De Munck J, Yoshida Y, Inoue S, Vargas M, Vijay P, et al. Buonocore memorial lecture. Adhesion to enamel and dentin: Current status and future challenges. Oper Dent 2003; 28(3):215-35.

4. Carvalho RM, Chersoni S, Frankenberger R, Pashley DH, Prati C, Tay FR. A challenge to the conventional wisdom that simultaneous etching and resin infiltration always occurs in self-etch adhesives. Biomaterials 2005; 26(9):1035-42.

5. Yoshida Y, Nagakane K, Fukuda R, Nakayama Y, Okazaki M, Shintani H, et al. Comparative study on adhesive performance of functional monomers. J Dent Res 2004; 83(6):454-8.

6. Bayne SC, Thompson JY, Swift Jr EJ, Stamatiades P, Wilkerson M. A characterization of first-generation flowable composites. J Am Dent Assoc 1998; 129(5):567-77.

7. Fu J, Kakuda S, Pan F, Hoshika S, Ting S, Fukuoka A, et al. Bonding performance of a newly developed step-less allin-one system on dentin. Dent Mater J 2013; 32(2):203-11.
8. Garcia RN, Morelli AE, Silva BS, Giongo BM, Pollheim CP, Largura GS, et al. Bonding performance of a self-adhering flowable composite to substrates used in direct technique. RSBO 2013; 10(4):343-9.

9. Garcia RN, Silva CS, Maisonnette FO, Silva GG, Mocellin $\mathrm{G}$, Ozelame J, et al. Bonding performance of a self-adhering flowable composite to indirect restorative materials. RSBO 2014; 11(1):6-12.

10. Shimada Y, Yamaguchi S, Tagami J. Microshear bond strength of dual-cured resin cement to glass ceramics. Dent Mater 2002; 18(5):380-8.

11. Versluis A, Tantbirojn D, Douglas WH. Why do shear bond tests pull out dentin? J Dent Res 1997; 76(6):1298-307.

12. Sano H, Shono T, Sonoda H, Takatsu T, Ciucchi B, Carvalho RM. Relation between surface area for adhesion and tensile bond strength - evaluation of a microtensile bond test. Dent Mater 1994; 10(4):236-40.

13. Holzmeier M, Schaubmayr M, Dasch W, Hirschfelder U. A new generation of self-etching adhesives: Comparison with traditional acid etch technique. J Orofac Orthop 2008; 69(2):78-93.

14. Erickson RL, Barkmeier WW, Kimmes NS. Bond strength of self-etch adhesives to pre-etched enamel. Dent Mater 2009; 25(10):1187-94.

15. Goracci C, Bertelli E, Ferrari M. Bonding to worn or fractured incisal edges: Shear bond strength of new adhesive systems. Quintessence Int 2004; 35(1):21-7.

16. Loguercio AD, Moura SK, Pellizzaro A, Dal-Bianco K, Patzlaff RT, Grande RH, et al. Durability of enamel bonding using two-step self-etch systems on ground and unground enamel. Oper Dent 2008; 33(1):79-88.

17. Perdigão J, Gomes G, Duarte S Jr, Lopes MM. Enamel bond strengths of pairs of adhesives from the same manufacturer. Oper Dent 2005; 30(4):492-9.

18. Peumans M, Munck J, Van Landuyt K, Lambrechts P, Van Meerbeek B. Three-year clinical effectiveness of a two-step self-etch adhesive in cervical lesions. Eur J Oral Sci 2005; 113(4):512-8.

19. Benetti P, Fernandes VV, Torres CR, Pagani C. Bonding efficacy of new self-etching, self-adhesive dual-curing resin cements to dental enamel. J Adhes Dent 2011; 13(3):231-4.

20. Lima AF, da Silva VB, Soares GP, Marchi GM, Baggio Aguiar $\mathrm{FH}$, Lovadino JR. Influence of previous acid etching on interface morphology and bond strength of self-etching adhesive to cavosurface enamel. Eur J Dent 2012; 6(1):56-62.

21. Watanabe T, Tsubota K, Takamizawa T, Kurokawa H, Rikuta A, Ando S, et al. Effect of prior acid etching on bonding durability of single-step adhesives. Oper Dent 2008; 33(4):426-33.

22. Poitevin A, De Munck J, Van Ende A, Suyama Y, Mine A, Peumans M, et al. Bonding effectiveness of self-adhesive composites to dentin and enamel. Dent Mater 2013; 29(2):221-30.

23. Di Hipólito V. Efeito de sistemas adesivos sobre a superfície do esmalte dental íntegro e desgastado [Dissertação de Mestrado]. Piracicaba: Faculdade de Odontologia de Piracicaba, Universidade Estadual de Campinas; 2005.

24. Garberoglio R, Brännström M. Scanning electron microscopic investigation of human dentinal tubules. Arch Oral Biol 1976; 21(6):355-62

25. Swift Junior EJ, Perdigão J, Heymann HO. Bonding to enamel and dentin: a brief history and state of the art. Quintessence Int 1995; 26(2):95-110.

26. Garcia RN, de Goes MF, Giannini M. Effect of water storage on bond strength of self-etching adhesives to dentin. $\mathrm{J}$ Contemp Dent Pract 2007; 8(7):46-53. 
27. Garcia RN, Reis AF, Giannini M. Effect of activation mode of dual-cured resin cements and low-viscosity composite liners on bond strength to dentin. J Dent 2007; 35(7):564-9.

28. Bektas OO, Eren D, Akin EG, Akin H. Evaluation of a selfadhering flowable composite in terms of micro-shear bond strength and microleakage. Acta Odontol Scand 2013; 71(34):541-6.

29. Teixeira EC, Bayne SC, Thompson JY, Ritter AV, Swift EJ. Shear bond strength of self-etching bonding systems in combination with various composites used or repairing aged composites. J Adhes Dent 2005; 7(2):159-64.

30. Menezes FC, Silva SB, Valentino TA, Oliveira MA, Rastelli AN, Gonçalves LS. Evaluation of bond strength and thickness of adhesive layer according to the techniques of applying adhesives in composite resin restorations. Quintessence Int 2013; 44(1):9-15.

\section{Endereço para correspondência:}

Rubens Nazareno Garcia

Rua Uruguai, 458

Centro

88302-202 Itajaí, SC

Telefone: (47) 99837416 / 91912522

E-mail: rubensgarcia@univali.br

Recebido: 21/01/16. Aceito: 20/06/2016. 